syndrome. The EEG was normal in 2 and at follow-up in one other whose initial record had shown focal temporal slow and sharp waves. Cases of AC involving the temporal lobe warrant psychological testing for associated ADHD and learning disorders.

\title{
CEREBELLAR VERMIS SPLIT SYNDROME
}

Surgical transection of the posterior inferior cerebellar vermis in 5 children (ages 6 - 15 years) treated at Washington University School of Medicine, St Louis, MO, was followed by selective impairment of tandem gait. Surgery performed for removal of tumors in the fourth ventricle involved destruction of the midline parallel fibers ranging from lobules VI-X. Regular self-paced gait, Romberg posture, and hopping were only minimally impaired, and kicking, reaching, or speech were unaffected. (Bastian AJ, Mink JW, Kaufman BA, Thach WT. Posterior vermal split syndrome. Ann Neurol Oct 1998;44:601-610). (Respond: Dr Bastian. Program in Physical Therapy, Washington University School of Medicine, 4444 Forest Park Parkway, Box 8502, St Louis, MO 63108).

COMMENT. Profound and persistent ataxia of tandem gait occurs after surgical division of midline fibers crossing the posterior inferior vermis of the cerebellum, whereas regular gait, hopping, individual limb coordination, and speech are relatively unaffected. Damage to both vestibular and visual inputs subserved by the posterior midline vermis fibers may explain the preferential impairment of tandem gait.

Vermian agenesis without posterior fossa cyst or fourth ventricle enlargement was diagnosed by MRI at 16 months to 5 years in 14 children presenting with oculo-motor apraxia, ataxia, and global developmental delay at Saint-Vincent de Paul Hospital, Paris, France (Nabbout R, Bulteau C et al. Brain Dev Sept 1998;20:429), and presented at the VIII International Child Neurology Congress, Ljubljana, Slovenija, Sept 1998.

\section{SEIZURE DISORDERS}

\section{EFFICACY OF KETOGENIC DIET AGAIN RE-VISITED}

A prospective multicenter study of the efficacy of the ketogenic diet in treatment of 51 children with intractable seizures is reported from 6 large institutions, including Johns Hopkins, Montefiore and Boston, USA, and Halifax, Canada, and 1 private practice setting. A 4:1 ketogenic diet was introduced in hospitalized patients after 36 hours fasting, according to the Johns Hopkins protocol. Pre-diet parental estimates of seizure frequencies were compared with seizure occurrences after 3, 6, and 12 months treatment. A 50\% decrease in seizure frequency or greater was observed in $54 \%$ at 3 months, in $55 \%$ at 6 months, and in $40 \%$ at 1 year. Only 5 patients were seizure-free at 1 year. The fall out rate of patients starting the diet was $12 \%$ at 3 months, $31 \%$ at 6 months, and $53 \%$ at 1 year. Reasons for discontinuance of the diet were medical intolerance due to lethargy, dehydration, vomiting, or behavioral problems in 6 , the restrictive dietary protocol in 4, and lack of seizure control in 12. Seizure type (tonic-clonic in 20, myoclonic (19), atonic (14), absence (8), and partial (9)) and EEG abnormality (generalized or multifocal epileptiform) were not related to outcome. (Vining EPG, Freeman JM, Ballaban-Gil K, et al, and the Ketogenic Diet Multi-Center Study Group. A multicenter study of the efficacy of the ketogenic diet. Arch Neurol Nov 1998;55:1433-1437). (Respond: Eileen PG Vining MD, The Johns Hopkins Medical 
Institutions, Meyer 2-147-JHH, $600 \mathrm{~N}$ Wolfe St, Baltimore, MD 21287).

COMMENT. That the ketogenic diet (KD) works has been established and corroborated by numerous investigators in the past. Is it of practical value in the management of childhood epilepsy is perhaps a more pressing question, given the advent of newer antiepileptic drugs? The above multicenter study, completed primarily in university settings, corroborates once again the partial efficacy of the KD, when introduced under hospitalized supervision, and when patients are followed closely by dietary, nursing, and physician services. Antiepileptic medication must be continued in the majority of patients treated, compounding the cost of dietary services, frequently not covered by health insurance plans.

Despite close supervision, complete control of seizures may be expected in only $10 \%$ of patients and $53 \%$ will have discontinued the diet after one year. Medical intolerance, the restrictive regimen, and insufficient seizure control to justify the demands of the diet are reasons for its withdrawal. Serious side effects, not mentioned in the above report, are the subject of a paper recently published by one of the participant authors and institutions (Ballaban-Gil $\mathrm{K}$ et al. Complications of the ketogenic diet. Epilepsia July 1998;39:744-748; see Ped Neur Briefs Aug 1998;12:6()). In $5(10 \%)$ of 52 children treated with the KD, adverse events included hypoproteinemia, lipemia, hemolytic anemia, and Fanconi's renal tubular acidosis; two patients had severe abnormalities of liver function, one with concomitant thrombocy topenia.

The ketogenic diet may be of value as a short-term therapy in a limited group of children with refractory epilepsy or in those exhibiting serious adverse reactions to AEDs. Its use should perhaps be restricted to research institutions where studies of the mechanism of action of the diet might uncover an essential specific ingredient and further our understanding of seizure pathophysiology (see Progress in Pediatric Neurology, PNB Publ, Vol I, 1991;pp 87-88; Vol III, 1997;pp578-9). To paraphrase the conclusions of an editorial (Roach ES. Alternative neurology. The ketogenic diet. Arch Neurol Nov 1998;55:1403-4), "Those who champion alternative treatments have an obligation to study them thoroughly before promoting them for general use with patients." "They should also be accountable for the cost-effectiveness of their methods." The Mayo Clinic KD protocol, which avoids the necessity for pre-diet starvation and hospitalization of the patient, is in my experience more practical and cost-effective and equally successful (Millichap JG, Jones JD, Rudis BP. Mechanism of the anticonvulsant action of the ketogenic diet. Am I Dis Child 1964;107:593).

\section{BONE MINERAL DENSITY UNAFFECTED BY ANTIEPILEPTICS}

The effects of valproic acid (VPA) and carbamazepine (CBZ) on bone mineral density were evaluated in 53 children with primary epilepsy treated for longer than 1 year at the Gulhane Military Academy, Ankara, Turkey. Mean serum levels of VPA and CBZ were 66 and $7 \mathrm{mcg} / \mathrm{ml}$, respectively. Serum levels of calcium and phosphorus were normal. Mineral densities measured by x-ray absorptiometry at L2-L4 were not significantly different from a control group. (Akin R, Okutan V, Sarici U et al. Evaluation of bone mineral density in children receiving antiepileptic drugs. Pediatr Neurol Aug 1998;19:129-131). (Respond: R Akin MD, Department of Pediatrics, Gulhane Military Medical Academy, Ankara, Turkey).

COMMENT. Previous studies have referred to the occurrence of rickets in children taking antiepileptic drugs. A decrease in serum calcium and elevations in alkaline phosphatase (ALP) have been reported, and nonambulatory, handicapped children are at greater risk of rickets. Elevated ALP values, observed 